\title{
To Eat or Not to Eat-An Inflammatory Issue
}

\section{Jenna RM*}

School of Life Sciences, University of Sussex, UK

Mini Review

Volume 1 Issue 1

Received Date: July 19, 2018

Published Date: August 13, 2018

DOI: $10.23880 /$ aabsc- 16000103

JMS Building, University of Sussex, Falmer, UK, Email: j.macciochi@sussex.ac.uk

\section{Abstract}

Comprised of a rich and complex microbial ecosystem, the human intestinal tract is both an immunological organ and supplier of the body's energy demand and nutrient requirements. This intestinal microbiota provides a large reservoir of bacterial lipopolysaccharide, (known also as endotoxin) that has an immediate stimulatory action on the innate immune system. The gut has evolved mechanisms to detoxify endotoxin and neutralise its potential inflammatory properties. However, this potent inflammatory molecule is transiently detectable in the circulation of healthy individuals following ingestion of food by virtue of a transient gut epithelial permeability arising from the digestive process itself. This acute post-prandial inflammation is somewhat dependent on meal composition with energy rich meals dense in saturated fat and low in fibre and polyphenols exacerbating the process. Chronic exposure to circulating endotoxin by this mechanism has been associated with a dysregulated cardiometabolic phenotype and risk of cardiovascular disease. Spending the majority of wake time in the post-prandial state therefore may contribute to the pathogenesis of these diseases. In this review, I present an overview of the mechanisms by which post-prandial inflammatory events and raise the possibility of modulating meal frequency as a dietary tool to, at least in part, ameliorate the detrimental outcomes of endotoxemia.

Keywords: Inflammation; Epithelial Permeability; Endotoxin; Cardiovascular Disease; Metabolic Syndrome; Microbiome

\section{Introduction}

Designed to facilitate digestive function, the gastrointestinal tract forms the largest mucosal interface of the body with the outside world, supplying the body's energy demand and nutrient requirements. However, this anatomical set-up exposes the intestine to a variety of physiological and antigenic challenges. On the one hand, an intact intestinal barrier protects the human organism against invasion of microorganisms and toxins, on the other hand, this barrier must be open to absorb essential fluids and nutrients. Such opposing goals are achieved only by the complex anatomical and functional structure of the intestinal barrier. The term 'intestinal permeability', also known as leaky gut, refers to the functional status of this barrier. The intestinal barrier also has a long history as an immunological organ, hosting more immune cells than any other location in our bodies [1]. Living alongside a microbial ecosystem, these potential combatants are actually collectively responsible for a wide array of critical immune-regulatory tasks. Thus, the intestinal barrier is complex and multi-layer both physical and functional, immune and microbial. 


\section{Falling Through the Cracks}

Appropriate fluctuations in permeability of the intestinal epithelium is a functional feature crucial for the balance between nutrient absorption during ingestion of a meal and microbe exclusion from the underlying lamina propria. A scaffold of tight junctions (TJ) including zonulins, occludins, claudins and junctional adhesion molecules modulate movement from intestinal lumen to bloodstream. Digestion itself negatively affects TJ assembly, increasing gut permeability and permitting the transient presence of low doses of pro-inflammatory bacterial compounds into the blood, termed endotoxemia caused by Lipopolysaccharide (LPS, also known as endotoxin), a component of the gram-negative bacterial cell wall translocating the epithelial barrier. Endotoxin has an immediate impact on the activation of the distil innate immune system via Toll-like receptors leading to intracellular activation of nuclear factor factor-?B (NF[3), systemic inflammatory cytokine release (including Tumour Necrosis Factor-? - TNF?, Interleukin-1 - IL-1 and interleukin-6 IL-6) which participate in the pathogenesis of epithelial barrier dysfunction and trigger release of acute phase proteins (including C-reactive protein - CRP and Serum Amyloid A - SAA) by the liver $[2,3]$. The mechanisms of these postprandial inflammatory responses are exaggerated by lipaemia and increased chylomicron formation and triacylglyceride (TAG) content in circulation. Chylomicron and TAG adherence to, and activation of, monocytes further provokes the immune response $[4,5]$. Interestingly, TAG levels increase from baseline in a similar pattern to endotoxin over a four-hour post-prandial period but despite higher baseline, TAG levels did not increase significantly in metabolically compromised over healthy individuals.

\section{Acute by Design}

Digestion in and of itself is an inflammatory process producing endotoxemia and low-grade inflammation by virtue of epithelial permeability and microbial products translocating to the blood where they switch on inflammatory events [6]. Inflammation is acute by design. In healthy adults, the majority of post-prandial inflammatory cytokine events are short-lived, detected as early as one hour after a meal and remaining elevated for up to four hours $[7,8]$. Acute phase SAA and CRP follow quite different kinetics with almost no detectable increase up to five hours before a slow rise to peak at around 24 hours. While this is a normal physiological phenomenon, an energy rich 'western-style' diet significantly increases this mechanism and data are now accumulating that emphasise the importance of epithelial barrier integrity to minimising LPS translocation to overall health $[9,10]$. High fat intake has also received particular attention in induction of epithelial permeability. Not only do high fat diets encourage gram negative bacteria at the expense of gram positive, but endotoxin has a strong affinity for chylomicrons (lipoproteins that transport dietary lipids including long-chain saturated fatty acids (SFAs) through the gut wall) and, as such, can cross the gastrointestinal mucosa coupled with damaging lipoproteins [11,12]. Thus, fat intake may induce endotoxemia via enhanced intestinal absorption related to chylomicron formation. However, it has also been suggested that total energy intake, rather than fat intake per se, confounds these previous observations [13]. The 'leaky gut hypothesis' explains how frequent or inappropriate intestinal barrier permeability is associated with the incidence of disease including cardiometabolic diseases, type two diabetes and other inflammatory and autoimmune syndromes [14,15]. Efforts to identify sources elevating systemic inflammatory tone have implicated gut-derived endotoxin as a trigger [7]. This has led to the common assumption that such conditions may reflect a chronic low-level LPS driven activation of the systemic innate immune system $[16,17]$. Unlike higher doses of serum LPS such as those seen in the case of sepsis, post-prandial low level circulating endotoxin has little impact on malaise and temperature while retaining more subtle inflammatory features [18-20]. It should be borne in mind that the majority of studies evaluating endotoxemia are done in the fasted state thus the kinetics of experimentally induced endotoxemia could differ from the post-prandial physiological state coming from a meal. In addition, means used to assess permeability can vary widely and have been reviewed elsewhere $[9,21]$.

\section{Regulation by Mouth \& Microbes}

To counter the various dietary barrier disruptors, extensive epithelial TJ networks are dynamically regulated by a diverse array of protective factors many of which recruit the use of the cacophony of bio-active compounds in the diet, the microbiome themselves and their metabolites. Short chain fatty acids (SCFA), the microbial fermentation products of dietary fibre are one well documented example $[22,23]$. These end-products of microbial fermentation facilitate tight junction assembly and stimulate the production of intestinal alkaline phosphatase involved in regulation of chylomicron transport and detoxification of endotoxins [24,25]. More recently, Vitamin $D$ has been shown to modulate tight junctions [26]. Finally, polyphenols, natural plant derived metabolites known to exert various anti-inflammatory 
and antioxidant benefits, as recently shown by Yang et al. to have a beneficial effect on the epithelial barrier integrity [27]. Polyphenols are considered to exert their protective effects on the epithelial barrier by targeting different members of the NF-?B family and antagonising subsequent pro-inflammatory cytokine production that cause TJ disassembly. In vitro, naringenin, a major polyphenol in citrus fruits and curcumin, a major polyphenol in turmeric enhanced tight junction formation and barrier integrity and in vivo, countered chemically induced gut epithelial damage [28-30]. Grape seed extract (GSE) that contains a mixture of polyphenols reduced endotoxemia in experimentally induced compromised barrier integrity and promoted the mRNA expression of TJ proteins [31]. In addition, resveratrol, a polyphenol extracted from grape seed and skin, promotes the mRNA expression of TJ proteins [32]. The role of NF- $\kappa B$ in the impairment of the intestinal barrier is further supported by treatment with the NF- $\mathrm{BB}$ inhibitor pyrolidinedithiocarbamate [33]. Piegholdt reported the isoflavonebiochaninA improved TEER in NF- $\mathrm{KB} / \mathrm{TNF}-$ $\alpha$ induced disruption of barrier integrity in vitro [34]. Sirtuin 1 (SIRT1) is a $\mathrm{NAD}^{+}$-dependent protein deacetylate that sense environmental stress to alter intestinal integrity. SIRT1 has recently gained attention due to its association with increased longevity and as a protective upregulated by fasting [35]. Resveratrol, an agonist of SIRT1, alleviates TNF- $\alpha$ induced TJ Zonulin-1 disturbances leading to epithelial permeability in the gut [36].

\section{Meal Frequency as a Health Modulator}

Data assessing reduced meal frequency without calorie restriction have found little or conflicting impact on traditional cardiometabolic parameters such as body weight, plasma lipids or glucoregulatory factors $[37,38]$. Moving beyond traditional cardiometabolic parameters to the inflammatory process itself has only recently garnered significant investigative support. Consequently, there is a paucity of information on the effects of dietary intervention to markers of inflammation in cardiometabolic patients [39]. Considering low-grade inflammation may be acting upstream of traditionally assessed cardiometabolic risk factors, modification of meal frequency has potential to make an immediate and easily implementable clinical management tool particularly for obesity where current therapeutics are limited and offer only modest improvements. Obese women who changed their diet from multiple daily meals to alternate-day restriction exhibited significant reductions in levels of circulating TNF $\alpha$ and IL-6 [40]. However, moving upstream to inflammatory markers such as IL-6 and CRP is also complicated by ongoing controversy regarding their causal role and challenges in measurement due to circadian variation and dietary postprandial effects [41-43]. With the knowledge that metabolically 'at risk' individuals show exacerbated endotoxaemic responses, advising more frequent meals, even if they are smaller, has potential to allow endotoxin to spike several times per day thus precipitating lowgrade inflammation without desensitisation. Resulting downstream production of inflammatory and acute phase molecules would be in continuous production, as previous in vivo and in vitro studies have demonstrated [44-49]. This cumulate effect may actually exacerbate their inflammatory risk regardless of dietary content or energy balance but would be further aggravated in those consuming a high fat, low fibre/polyphenol content with gut dysbiosis.

Although known for quite some time, post-prandial epithelial permeability and consequential endotoxemia has yet to translate into a dietetic approach. The UK National Institute for Health and Care Excellence (NICE) advise a Mediterranean style diet based on a body of epidemiological, physiological and observational evidence demonstrating that such changes in diet are associated with reductions in morbidity and mortality from cardiovascular disease [50,51]. Current guidelines on meal frequency are often entangled with calorie reduction strategies [52]. For example, current recommendations for type 2 diabetics is to consume five small meals a day, presumed as a strategy to reduce hunger and therefore total energy intake although there are no clear insights into the supporting evidence. Kahleova et al. reported eating two larger meals as more effective than six smaller meals [53]. Observational trials in human indicate that eating more frequently than three times per day may play a role in obesity [54]. Other studies stress the division of food intake should be frequent but based on individual preference, with no clear recommendations on number of meals. As new data now demonstrates, society has crept towards a continual snacking routine and we now spend the majority of waking time in a post-prandial state [55]. Understanding the contribution of meal frequency and its acute and cumulative effects on inflammatory risk therefore warrants further investigation since leaving frequency to preference may not be beneficial, particularly in at risk populations.

Though studies have correlated increased eating frequency with increased body weight, what is not yet clear is how spending significant periods in the postprandial state impacts metabolic risk in healthy adults cumulatively over a long-period of time $[56,57]$. What we 
do know is that reduced frequency of eating through intermittent fasting (IF) and time-restricted eating (TRE) is an important aspect of eating frequency that claims to have broad effects on human health span and offers an innovative strategy to prevent and treat metabolic disease [58,59]. Restricting the food improves metabolic profiles but data from humans are limited. Mice under timerestricted feeding given equivalent energy intake from a high fat diet as those with ad libitum access are protected against obesity and metabolic abnormalities [60,61]. In addition, eating aligned with the diurnal circadian pattern of humans attenuates metabolic disease arising from a variety of obesogenic diets proportional to fasting duration and maintained even when temporarily interrupted [58]. Cyclical changes in the gut microbiome resulting from diurnal feeding and fasting rhythms contribute to the diversity of gut microflora and represent a mechanism by which the gut microbiome affects host metabolism. An extended fasting period (i.e., gut rest) could also lead to reduced gut permeability and upregulation of SIRT1, as a result, blunt postprandial endotoxemia and systemic inflammation [62-64]. Whether via IF, TRE or reducing the number of meals, we cannot ignore that dietary eating patterns impact health and disease [65]. Spending a large portion of time outwith the post-prandial state offers both a potential preventative and therapeutic intervention against diverse cardio-metabolic health challenges. Diet is not the only factor to cause barrier permeability and barrier permeability is not the only contributor to low grade systemic inflammation [66]. An individual's risk of inflammatory disease result from the interplay of many factors including social, environmental and genetic contributions. At a time when inflammation is a unifying theme of the many lifestyle and age related diseases that are rapidly increasing in prevalence, medicine is moving toward modifiable lifestyle-based paradigm.

\section{Conclusion}

History is teaching us that it is an unhelpful reductionist tendency neither to pit individual causative dietary constituents against each other nor to discount the medications and procedures used as treatments. Ultimately, a meal is the sum of its parts, by studying centrality of dietary patterns and behaviours, away from isolated nutrient and energy intake, the minutia of individual dietary constituents become less relevant. While the role for saturated fat in cardiovascular disease remains heavily debated, there is no doubt that saturated fat can exacerbate dietary perturbations to the endothelium further facilitating LPS transfer via chylomicron formation [67-76]. Nor is there any dispute over continuing to promote the key role of lifestyle interventions such as regular exercise and consuming a fibre, nutrient and polyphenol dense diet for good cardiometabolic health and reducing unruly inflammation. But as the majority moving towards spending the majority of waking time in a post-prandial state cumulatively promote the inflamed state, reduced meal frequency may be preventative in nature with the benefits accruing over lifespan rather than prescribing a change in eating pattern after a diagnosis.

\section{Acknowledgement}

I am grateful to my colleague current and past and the many clinicians promoting lifestyle medicine in the UK.

\section{References}

1. Peterson LW, Artis D (2014) Intestinal epithelial cells: regulators of barrier function and immune homeostasis. Nat Rev Immunol 14(3): 141-153.

2. McKenzie JA, Ridley AJ (2007) Roles of Rho/ROCK and MLCK in TNF-alpha-induced changes in endothelial morphology and permeability. J Cell Physiol 213(1): 221-228.

3. Clemente-Postigo $M$, Queipo-Ortuño MI, Murri $M$, Boto-Ordoñez M, Perez-Martinez P, et al. (2012) Endotoxin increase after fat overload is related to postprandial hypertriglyceridemia in morbidly obese patients. J Lipid Res 53(5): 973-978.

4. Candore G, Colonna-Romano G, Balistreri CR, Di Carlo D, Grimaldi MP, et al. (2006) Biology of longevity: role of the innate immune system. Rej Res 9(1): 143-148.

5. Klop B, Proctor SD, Mamo JC, Botham KM, Castro Cabezas M (2012) Understanding postprandial inflammation and its relationship to lifestyle behaviour and metabolic diseases. Int J Vas Med.

6. Kvietys PR, Specian RD, Grisham MB, Tso P (1991) Jejunal mucosal injury and restitution: role of hydrolytic products of food digestion. Am J Physiol 261(3): G384-G391.

7. Herieka M, Erridge C (2014) High-fat meal induced postprandial inflammation. Mol Nutr Food Res 58(1): 136-146.

8. Nádházi Z, Takáts A, Offenmüller K, Bertók L (2002) Plasma endotoxin level of healthy donors. J Microbiol Immunol Hungary 49(1): 151-157. 
9. Pendyala S, Walker JM, Holt PR (2012) A high-fat diet is associated with endotoxemia that originates from the gut. Gastroenterology 142(5): 1100-1101.

10. Rahman K, Desai C, Iyer SS, Thorn NE, Kumar P, et al. (2016) Loss of Junctional Adhesion Molecule A Promotes Severe Steatohepatitis in Mice on a Diet High in Saturated Fat, Fructose, and Cholesterol. Gastroenterology 151(4): 733-746.

11. Cani PD, Neyrinck AM, Fava F, Knauf C, Burcelin RG, et al. (2007) Selective increases of bifidobacteria in gut microflora improve high-fat-diet-induced diabetes in mice through a mechanism associated with endotoxaemia. Diabetologia 50(11): 2374-2383.

12. Kelly CJ, Colgan SP, Frank DN (2012) Of microbes and meals: the health consequences of dietary endotoxemia. Nutr Clin Practice 27(2): 215-225.

13. Amar J, Burcelin R, Ruidavets JB, Cani PD, Fauvel J, et al. (2008) Energy intake is associated with endotoxemia in apparently healthy men. Am J Clin Nutr 87(5): 1219-1223.

14. Fändriks L (2017) Roles of the gut in the metabolic syndrome: an overview. J Int Med 281(4): 319-336.

15. Mu Q, Kirby J, Reilly CM, Luo XM (2017) Leaky Gut as a Danger Signal for Autoimmune Diseases. Front Immunol 8: 598.

16. Erridge C, Attina T, Spickett CM, Webb DJ (2007) A high-fat meal induces low-grade endotoxemia: evidence of a novel mechanism of postprandial inflammation. Am J Clin Nutr 86(5): 1286-1292.

17. Cani PD, Amar J, Iglesias MA, Poggi M, Knauf C, et al. (2007) Metabolic endotoxemia initiates obesity and insulin resistance. Diabetes 56(7): 1761-1772.

18. Starkie R, Ostrowski SR, Jauffred S, Febbraio M, Pedersen BK (2003) Exercise and IL-6 infusion inhibit endotoxin-induced TNF-alpha production in humans. FASEB J 17(8): 884-886.

19. Krogh-Madsen R, Plomgaard P, Akerstrom T, Møller K, Schmitz O, et al. (2008) Effect of short-term intralipid infusion on the immune response during low-dose endotoxemia in humans. Am J Phys Endo Metab 294(2): E371-E379.

20. Krogh-Madsen R, Møller K, Dela F, Kronborg G, Jauffred S, et al. (2004) Effect of hyperglycemia and hyperinsulinemia on the response of IL-6, TNF-alpha, and FFAs to low-dose endotoxemia in humans. Am J Phys Endo Metab 286(5): E766-E772.

21. Taudorf S, Krabbe KS, Berg RM, Pedersen BK, Møller K (2007) Human models of low-grade inflammation: bolus versus continuous infusion of endotoxin. Clinical and Vaccine Immunology 14(3): 250-255.

22. Cani PD, Bibiloni R, Knauf C, Waget A, Neyrinck AM, et al. (2008) Changes in gut microbiota control metabolic endotoxemia-induced inflammation in high-fat diet-induced obesity and diabetes in mice. Diabetes 57(6): 1470-1481.

23. Ohata A, Usami M, Miyoshi M (2005) Short-chain fatty acids alter tight junction permeability in intestinal monolayer cells via lipoxygenase activation. Nutrition 21(7-8): 838-847.

24. Peng L, Li ZR, Green RS, Holzman IR, Lin J (2009) Butyrate enhances the intestinal barrier by facilitating tight junction assembly via activation of AMP-activated protein kinase in Caco-2 cell monolayers. J Nutr 139(9): 1619-1625.

25. Lallès JP (2010) Intestinal alkaline phosphatase: multiple biological roles in maintenance of intestinal homeostasis and modulation by diet. Nutr Rev 68(6): 323-332.

26. Stio M, Retico L, Annese V, Bonanomi AG (2016) Vitamin D regulates the tight-junction protein expression in active ulcerative colitis. Scandinavian Journal of Gastroenterology 51(10): 1193-1199.

27. Yang G, Bibi S, Du M, Suzuki T, Zhu MJ (2017) Regulation of the intestinal tight junction by natural polyphenols: A mechanistic perspective. Crit Rev Food Sci Nutr 57(18): 3830-3839.

28. Noda S, Tanabe S, Suzuki T (2012) Differential effects of flavonoids on barrier integrity in human intestinal Caco-2 cells. J Agri Food Chem 60: 4628-4633.

29. Wang N, Wang G, Hao J, Ma J, Wang Y (2012) Curcumin ameliorates hydrogen peroxide-induced epithelial barrier disruption by upregulating heme oxygenase-1 expression in human intestinal epithelial cells. Dig Dis Sci 57(7): 1792-1801.

30. Azuma T, Shigeshiro M, Kodama M, Tanabe S, Suzuki $\mathrm{T}$ (2013) Supplemental naringenin prevents intestinal barrier defects and inflammation in colitic mice. J Nutr 143(6): 827-834. 
31. Cheah KY, Bastian SE, Acott TM, Abimosleh SM, Lymn KA, et al. (2013) Grape seed extract reduces the severity of selected disease markers in the proximal colon of dextran sulphate sodium-induced colitis in rats. Dig Dis Sci 58(4): 970-977.

32. Etxeberria U, Arias N, Boqué N, Macarulla MT, Portillo MP, et al. (2015) Reshaping faecal gut microbiota composition by the intake of trans-resveratrol and quercetin in high-fat sucrose diet-fed rats. J Nutr Biochem 26(6): 651-660.

33. Yin J, Wu M, Duan J, Liu G, Cui Z, et al. (2015) Pyrrolidine Dithiocarbamate Inhibits NF-KappaB Activation and Upregulates the Expression of Gpx1, Gpx4, Occludin, and ZO-1 in DSS-Induced Colitis. Appl Biochem Biotechnol 177(8): 1716-1728.

34. Piegholdt S, Pallauf K, Esatbeyoglu T, Speck N, Reiss K, et al. (2014) Biochanin A and prunetin improve epithelial barrier function in intestinal CaCo-2 cells via downregulation of ERK, NF- $\kappa \mathrm{B}$, and tyrosine phosphorylation. Free Rad Biol Med 70: 255-264.

35. Pedersen SB, Ølholm J, Paulsen SK, Bennetzen MF, Richelsen B (2008) Low Sirt1 expression, which is upregulated by fasting, in human adipose tissue from obese women. Int J Obesity 32(8): 1250-1255.

36. Ma Y, Xu C, Wang W, Sun L, Yang S, et al. (2014) Role of SIRT1 in the protection of intestinal epithelial barrier under hypoxia and its mechanism. Chinese J Gastro Surgery 17(6): 602-606.

37. Varady KA (2016) Meal frequency and timing: impact on metabolic disease risk. Curr Op Endo Diab Obes 23(5): 379-383.

38. Mattson MP (2005) The need for controlled studies of the effects of meal frequency on health. Lancet 365(9475): 1978-1980.

39. Ridker PM (2016) From C-Reactive Protein to Interleukin-6 to Interleukin-1: Moving Upstream To Identify Novel Targets for Atheroprotection. Circulation Research 118(1): 145-156.

40. Kroeger CM, Klempel MC, Bhutani S, Trepanowski JF, Tangney CC, et al. (2012) Improvement in coronary heart disease risk factors during an intermittent fasting/calorie restriction regimen: Relationship to adipokine modulations. Nutrition \& Metabolism 9(1): 98.
41. Ridker PM, Rifai N, Stampfer MJ, Hennekens $\mathrm{CH}$ (2000) Plasma concentration of interleukin-6 and the risk of future myocardial infarction among apparently healthy men. Circulation 101(15): 1767-1772.

42. Ridker PM, Rifai N, Pfeffer M, Sacks F, Lepage S, et al. (2000) Elevation of tumor necrosis factor-alpha and increased risk of recurrent coronary events after myocardial infarction. Circulation 101(18): 21492153.

43. Ridker PM, Rose L, Cook NR. (2015) A proposal to incorporate trial data into a hybrid ACC/AHA algorithm for the allocation of statin therapy in primary prevention. J Am Coll Cardio 65(9): 942.

44. Youssef-Elabd EM, McGee KC, Tripathi G, Aldaghri N, Abdalla MS, et al. (2012) Acute and chronic saturated fatty acid treatment as a key instigator of the TLRmediated inflammatory response in human adipose tissue, in vitro. J Nutr Biochem 23(1): 39-50.

45. Creely SJ, McTernan PG, Kusminski CM, Fisher fM, Da Silva NF, et al. (2007) Lipopolysaccharide activates an innate immune system response in human adipose tissue in obesity and type 2 diabetes. American Journal of Physiology Endocrinology \& Metabolism 292(3): E740-E747.

46. Harte AL, da Silva NF, Creely SJ, McGee KC, Billyard T, et al. (2010) Elevated endotoxin levels in nonalcoholic fatty liver disease. J Inflammation 7: 15.

47. Harte AL, Varma MC, Tripathi G, McGee KC, Al-Daghri NM, et al. (2012) High fat intake leads to acute postprandial exposure to circulating endotoxin in type 2 diabetic subjects. Diabetes Care 35(2): 375382.

48. Ghanim H, Abuaysheh S, Sia CL, Korzeniewski K, Chaudhuri A, et al. (2009) Increase in plasma endotoxin concentrations and the expression of Tolllike receptors and suppressor of cytokine signaling-3 in mononuclear cells after a high-fat, highcarbohydrate meal: implications for insulin resistance. Diabetes Care 32(12): 2281-2287.

49. Deopurkar R, Ghanim H, Friedman J, Abuaysheh S, Sia CL, et al. (2010) Differential effects of cream, glucose, and orange juice on inflammation, endotoxin, and the expression of Toll-like receptor-4 and suppressor of cytokine signaling-3. Diabetes Care 33(5): 991-997. 
50. Wang X, Ouyang Y, Liu J, Zhu M, Zhao G, et al. (2014) Fruit and vegetable consumption and mortality from all causes, cardiovascular disease, and cancer: systematic review and dose-response meta-analysis of prospective cohort studies. BMJ 349: g4490.

51. Oyebode O, Gordon-Dseagu V, Walker A, Mindell JS (2014) Fruit and vegetable consumption and allcause, cancer and CVD mortality: analysis of Health Survey for England data. J Epidemiol Comm Health 68(9): 856-862.

52. St-Onge MP, Ard J, Baskin ML, Chiuve SE, Johnson HM, et al. (2017) Meal Timing and Frequency: Implications for Cardiovascular Disease Prevention: A Scientific Statement From the American Heart Association. Circulation. 135(9): e96-e121.

53. Kahleova H, Belinova L, Malinska H, Oliyarnyk O, Trnovska J, et al. (2014) Eating two larger meals a day (breakfast and lunch) is more effective than six smaller meals in a reduced-energy regimen for patients with type 2 diabetes: a randomised crossover study. Diabetologia 57(8): 1552-1560.

54. Howarth NC, Huang TT, Roberts SB, Lin BH, McCrory MA (2007) Eating patterns and dietary composition in relation to BMI in younger and older adults. International J Obesity 31(4): 675-684.

55. Gill S, Panda S (2015) A Smartphone App Reveals Erratic Diurnal Eating Patterns in Humans that Can Be Modulated for Health Benefits. Cell Metab 22(5): 789-798.

56. Ma Y, Bertone ER, Stanek EJ, Reed GW, Hebert JR, et al. (2003) Association between eating patterns and obesity in a free-living US adult population. American J Epidemiol 158(1): 85-92.

57. Bellisle F, McDevitt R, Prentice AM (1997) Meal frequency and energy balance. B J Nutr 77(S1): S57S70.

58. Chaix A, Zarrinpar A, Miu P, Panda S (2014) Timerestricted feeding is a preventative and therapeutic intervention against diverse nutritional challenges. Cell Metab 20(6): 991-1005.

59. Longo VD, Mattson MP (2014) Fasting: molecular mechanisms and clinical applications. Cell Metab 19(2): 181-192.

60. Hatori M, Vollmers C, Zarrinpar A, DiTacchio L, Bushong EA, et al. (2012) Time-restricted feeding without reducing caloric intake prevents metabolic diseases in mice fed a high-fat diet. Cell Meta 15(6): 848-860.

61. Sherman H, Genzer Y, Cohen R, Chapnik N, Madar Z, et al. (2012) Timed high-fat diet resets circadian metabolism and prevents obesity. FASEB J 26(8): 3493-3502.

62. Kelly CJ (2007) A controlled trial of reduced meal frequency without caloric restriction in healthy, normal-weight, middle-aged adults. Am J Clin Nutr 86(4): 1254-1255.

63. Moreira AP, Texeira TF, Ferreira AB, PeluzioMdo C, AlfenasRde C (2012) Influence of a high-fat diet on gut microbiota, intestinal permeability and metabolic endotoxaemia. Brit J Nutr 108(5): 801-809.

64. Tilg H, Kaser A (2011) Gut microbiome, obesity, and metabolic dysfunction. JCI 121(6): 2126-2132.

65. Zarrinpar A, Chaix A, Panda S (2016) Daily Eating Patterns and Their Impact on Health and Disease. Trends Endo Metabo 27(2): 69-83.

66. dePunder K, Pruimboom L (2015) Stress Induces Endotoxemia and Low-Grade Inflammation by Increasing Barrier Permeability. Front Immunol 6: 223.

67. Malhotra A, Redberg RF, Meier P (2017) Saturated fat does not clog the arteries: coronary heart disease is a chronic inflammatory condition, the risk of which can be effectively reduced from healthy lifestyle interventions. Brit J Sports Med 51(15): 1111-1112.

68. Eggesbø JB, Hjermann I, Lund PK, Joø GB, Ovstebø R, et al. (1994) LPS-induced release of IL-1 beta, IL-6, IL8, TNF-alpha and sCD14 in whole blood and PBMC from persons with high or low levels of HDLlipoprotein. Cytokine 6(5): 521-529.

69. Jackson KG, Delgado-Lista J, Gill R, Lovegrove JA, Williams CM, et al. (2012) The leptin receptor Gln223Arg polymorphism (rs1137101) mediates the postprandial lipaemic response, but only in males. Atherosclerosis 225(1): 135-141.

70. Laugerette F, Vors C, Géloën A, Chauvin MA, Soulage $\mathrm{C}$, et al. (2011) Emulsified lipids increase endotoxemia: possible role in early postprandial lowgrade inflammation. J Nutr Biochem 22(1): 53-59. 
71. Manning PJ, Sutherland WH, McGrath MM, de Jong SA, Walker RJ, et al. (2008) Postprandial cytokine concentrations and meal composition in obese and lean women. Obesity 16(9): 2046-2052.

72. Marques-Vidal P, Mazoyer E, Bongard V, Gourdy P, Ruidavets JB, et al. (2002) Prevalence of insulin resistance syndrome in southwestern France and its relationship with inflammatory and hemostatic markers. Diabetes Care 25(8): 1371-1377.

73. Patel C, Ghanim H, Ravishankar S, Sia CL, Viswanathan P, et al. (2007) Prolonged reactive oxygen species generation and nuclear factor-kappaB activation after a high-fat, high-carbohydrate meal in the obese. J Clin Endocrinol Metab 92(11): 44764479.

74. Russell RM (1992) Changes in gastrointestinal function attributed to aging. American Journal of Clin Nutrition 55(S6): S1203-S1207.

75. Saffray MJ (2014) Aging of the mammalian gastrointestinal tract: a complex organ system. Age 36(3): 9603.

76. vanDijk SJ, Mensink M, Esser D, Feskens EJ, Müller M, et al. (2012) Responses to high-fat challenges varying in fat type in subjects with different metabolic risk phenotypes: a randomized trial. PLoS One 7(7): e41388. 